\title{
Persistent Currents in Quantum Chaotic Systems
}

\author{
Shiro Kawabata \\ Physical Science Division, Electrotechnical Laboratory, 1-1-4 Umezono, Tsukuba, Ibaraki 305-8568, Japan
}

(August 4, 2018)

\begin{abstract}
The persistent current of ballistic chaotic billiards is considered with the help of the Gutzwiller trace formula. We derive the semiclassical formula of a typical persistent current $I^{\text {typ }}$ for a single billiard and an average persistent current $\langle I\rangle$ for an ensemble of billiards at finite temperature. These formulas are used to show that the persistent current for chaotic billiards is much smaller than that for integrable ones. The persistent currents in the ballistic regime therefore become an experimental tool to search for the quantum signature of classical chaotic and regular dynamics.
\end{abstract}

PACS numbers: 73.23.Ad, 03.65.Sq, 72.20.My

A charged particle moving in the presence of a vector potential acquires the Aharonov-Bohm $(\mathrm{AB})$ phase, leading to an interesting interference effect in a non-simply connected geometry. One of the most evident manifestations of this $\mathrm{AB}$ effect is the persistent current in a mesoscopic ring threaded by magnetic flux. Efforts in this direction haye been mostly concerned with the influence of disordere 10 and electron-electron interaction 10 on the persistent current. The quantum-mechanical magnetic properties of ballistic systems depends on the underlying classical dynamics. Therefore, the persistent current in these systems, i.e., billiards, is one of the most interesting models for studying quantum chap 11

Recently von Oppen and Riedel 12 showed that the typical semiclassical persistent current at zero temperature for single integrable billiards, $I^{t y p} \sim\left(k_{F} \ell\right)^{3 / 2}$, is larger than that for chaotic billiards, $I^{t y p} \sim k_{F} \ell$, by using dimensional analysis $\left(k_{F}\right.$ is the Fermi wave number, and $\ell$ is the typical dimension of the billiard ${ }^{\text {Based on these }}$ results, Richter, Ullmo, and Jalabert 23 calculated the average persistent current $\langle I\rangle$ for an ensemble made of integrable billiards with a dispersion in size or in the electron filling by using the Berry-Tabor formula, 14.15 and showed that it scales as $\langle I\rangle \sim k_{F} \ell$. Until now, however, explicit semiclassical formulas for persistent current in single chaotic billiards or an ensemble of chaotic billiards have not been derived.

In this paper, we study the persistent current for quantum chaptic systems by employing the Gutzwiller trace formula.16 We calculate $I$ at a finite temperature, and derive the formulas for both single billiards, $I^{t y p}$, and an ensemble of billiards, $\langle I\rangle$. These formulas are used to show that $I^{t y p} \sim k_{F} \ell$ and $\langle I\rangle \sim\left(k_{F} \ell\right)^{0}$. Furthermore, it turns out that the amplitude of $I$ for quantum chaotic systems is much smaller than that for quantum integrable systems. This means that one-parameter families of periodic orbits existing in integrable systems interfere constructively, and give a total contribution much larger than that of the unstable isolated periodic orbits typical of chaotic systems.
The persistent current, induced by the magnetic flux $\phi$ in the AB geometry (see Fig. 1), can be obtained from the Helmholtz free energy $F(T, \phi, N)$ as

$$
I=-c\left(\frac{\partial F}{\partial \phi}\right)_{T, N}
$$

The Helmholtz free energy $F(T, \phi, N)$ for a fixed number $N$ of electrons and the grand canonical thermodynamic potential $\Omega(T, \phi, \mu)$ are related by means of a Legendre transform

$$
F(T, \phi, N)=\mu N+\Omega(T, \phi, \mu),
$$

where $\mu$ is the chemical potential of a particle reservoir. $\Omega$ is given by

$$
\Omega(T, \phi, N)=-\frac{1}{\beta} \int d E d(E, \phi) \ln (1+\exp [\beta(\mu-E)]),
$$

where $d(E, \phi)$ is one-particle density of states and $\beta=$ $1 / k_{B} T$. Within the semiclassical approximation, $d(E, \phi)$ can be decomposed into mean (Weyl) and oscillating parts, i.e.,

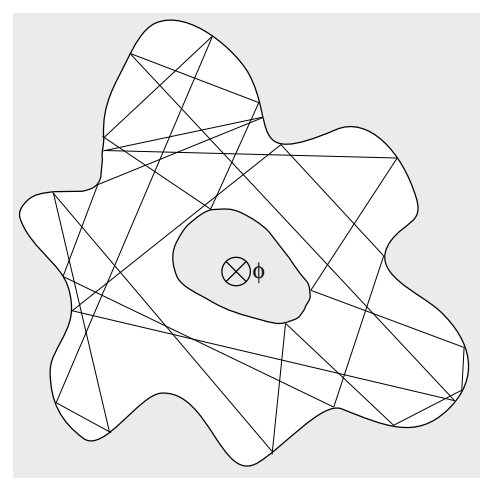

FIG. 1. A schematic illustration of a chaotic billiard with a rough boundary threaded by $\mathrm{AB}$ flux $\phi$. An example of an unstable periodic orbit is shown. 


$$
d(E)=d^{0}(E)+d^{o s c}(E, \phi) .
$$

The Weyl term depends on the classical phase volume and is independent of $\phi$. (The higher $\hbar$ corrections to $d^{0}$ give the standard bulk Landau magnetism 13$) d^{\text {osc }}(E)$ can be written as a sum over classical periodic orbits $s$,

$$
\begin{aligned}
d^{o s c}(E, \phi) & =\frac{1}{\pi \hbar} \sum_{s} \frac{\tau_{s}}{\left|\operatorname{det}\left(M_{s}-I\right)\right|^{1 / 2}} \\
& \times \cos \left(\frac{S_{s}}{\hbar}+2 \pi \omega_{s} \frac{\phi}{\phi_{0}}-\sigma_{s} \frac{\pi}{2}\right),
\end{aligned}
$$

where $\tau_{s}, M_{s}, S_{s}, \omega_{s}$, and $\sigma_{s}$ are the period, the monodromy matrix, the action integral, the winding number and Maslov index respectively. Following Richter, Ullmo, and Jalabert, 13 we define a mean chemical potential $\bar{\mu}$ by the condition of accommodating $\boldsymbol{N}$ electrons to the mean number of states,

$$
\boldsymbol{N}=N(\mu)=\bar{N}(\bar{\mu}) .
$$

Here

$$
N(\mu)=\int_{0}^{\infty} d E d(E) f(E-\mu),
$$

where $f(E-\mu)$ is the Fermi-Dirac distribution function. $\bar{N}(\bar{\mu})$ is obtained in eq (7) by replacing $d(E)$ and $\mu$ by $d^{0}(E)$ and $\bar{\mu}$. The Helmholtz free energy in terms of grand canonical quantities is obtained by expanding eq. (2) to second order in $\mu-\bar{\mu}$ as

$$
F(N) \approx F^{0}+\Delta F^{(1)}+\Delta F^{(2)},
$$

with

$$
\begin{aligned}
F^{0} & =\bar{\mu} \boldsymbol{N}+\bar{\Omega}(\bar{\mu}), \\
\Delta F^{(1)} & =\sum_{s} R_{T}\left(L_{s}\right)\left(\frac{\hbar}{\tau_{s}}\right)^{2} d^{o s c}(\bar{\mu}, \phi), \\
\Delta F^{(2)} & =\frac{1}{2 \bar{d}(\bar{\mu})}\left[\sum_{s} \frac{-R_{T}\left(L_{s}\right)}{\pi\left|\operatorname{det}\left(M_{s}-I\right)\right|^{1 / 2}}\right. \\
& \left.\times \sin \left(\frac{S_{s}}{\hbar}+2 \pi \omega_{s} \frac{\phi}{\phi_{0}}-\sigma_{s} \frac{\pi}{2}\right)\right]^{2},
\end{aligned}
$$

where $L_{s}=v_{F} \tau_{s}$ is the length of trajectory $s$, and $\bar{d}(\bar{\mu})=$ $m A / 2 \pi \hbar^{2}$ ( $m$ is the mass of electron and $A$ is the area of the billiard). In eqs. (10) and (11),

$$
R_{T}\left(L_{s}\right)=\frac{L_{s} / L_{c}}{\sinh \left(L_{s} / L_{c}\right)},
$$

with $L_{c}=\hbar v_{F} \beta / \pi$, yields an exponential suppression for the contribution of trajectories with $L>L_{c}$.

The leading-order contribution to $F$ is given by $F^{0}+$ $\Delta F^{(1)}$, and yields the persistent current of single chaotic billiard calculated in the grand canonical ensemble as

$$
\begin{aligned}
I^{(1)}(\phi) & =\frac{e}{\pi} v_{F} \sum_{s} \frac{R_{t}\left(L_{s}\right) \omega_{s}}{L_{s}\left|\operatorname{det}\left(M_{s}-I\right)\right|^{1 / 2}} \\
& \times \sin \left(k_{F} L_{s}+2 \pi \omega_{s} \frac{\phi}{\phi_{0}}-\sigma_{s} \frac{\pi}{2}\right),
\end{aligned}
$$

where $v_{F}=\hbar k_{F} / m$ is the Fermi velocity. Since the system is time reversal invariant at zero magnetic flux, each periodic orbit is associated with a time-reversed partner having exactly the same characteristics except for an opposite winding number. Grouping the time-reversal trajectories $t\left(\omega_{t}>0\right)$ in eq. (13), we have

$$
\begin{aligned}
I^{(1)}(\phi) & =\frac{2 e}{\pi} v_{F} \sum_{t} \frac{R_{t}\left(L_{t}\right) \omega_{t}}{L_{t}\left|\operatorname{det}\left(M_{t}-I\right)\right|^{1 / 2}} \\
& \times \cos \left(k_{F} L_{t}-\sigma_{t} \frac{\pi}{2}\right) \sin \left(2 \pi \omega_{t} \frac{\phi}{\phi_{0}}\right) .
\end{aligned}
$$

The persistent current for single billiards exhibits a $\phi_{0}$ periodicity and changes from diamagnetic to paramagnetic by changing $k_{F}$.

In order to characterize the typical value of the persistent current for single chaotic billiard, we define

$$
\left.I^{t y p} \equiv \sqrt{\left\langle I^{(1)}(\phi)^{2}\right\rangle}\right|_{\phi / \phi_{0}=1 / 4},
$$

where the average $\langle\cdots\rangle$ denotes an averaging over $k_{F}$ or some external parameter associated with the roughness of the boundary. Upon averaging, the off-diagonal terms vanish due to the widely fluctuating phases, while the diagonal term survives the averaging. Therefore we obtain

$$
\begin{aligned}
\left\langle I^{(1)}(\phi)^{2}\right\rangle & =\left(\frac{\sqrt{2} e v_{F}}{\pi}\right)^{2} \sum_{t} \frac{R_{T}^{2}\left(L_{t}\right) \omega_{t}^{2}}{L_{t}^{2}\left|\operatorname{det}\left(M_{t}-I\right)\right|} \\
& \times \sin ^{2}\left(2 \pi \omega_{t} \frac{\phi}{\phi_{0}}\right) .
\end{aligned}
$$

To evaluate the sum of $t$, we order the orbits by their length $L_{t}$ and use the Hannay and Ozorio de Almeida (H-OdA) sum rule, 17 i.e.,

$$
\sum_{t} \frac{1}{\left|\operatorname{det}\left(M_{t}-I\right)\right|} \cdots=\int_{0}^{\infty} \frac{d L}{L} \sum_{\omega=1}^{\infty} P_{L}(\omega) \cdots .
$$

Here $P_{L}(\omega)$ denotes the classical distribution of $\omega$ that an orbit is of length $L$ and is approximately given by Gaussian distribution, 18.19

$$
P_{L}(\omega)=\frac{1}{\sqrt{2 \pi \overline{\omega^{2}(L)}}} \exp \left(-\frac{\omega^{2}}{2 \bar{\omega}^{2}(L)}\right),
$$

with a variance increasing linearly with $L$, i.e.

$$
\overline{\omega^{2}(L)}=\alpha \frac{L}{L_{0}},
$$


where $\alpha$ is a system dependent constant, and $L_{0}$ is the length for the shortest periodic orbit.

In the following, we calculate $I^{\text {typ }}$ in high-temperature regimes, $T \geq T_{t h}, 20$ where $T_{t h}=\hbar v_{F} \pi / k_{B} L^{*}$ is the threshold temperature. ( $L^{*}$ is the characteristic length for which periodic orbits gan be taken as uniformly distributed in phase space.21) In this regime, the thermal fluctuation washes out structures on the energy scale of the mean energy level spacing $\Delta$, and only short orbits contribute effectively to the persistent current.22 In this case one may exploit the asymptotic form $R_{T}(L) \approx$ $2\left(L / L_{c}\right) \exp \left(-L / L_{c}\right)$. Substituting eqs. (17) and (18) into eq. (16), and using the Poisson summation formula, lead to

$$
\begin{gathered}
\left\langle I^{(1)}(\phi)^{2}\right\rangle=\left(\frac{2 e v_{F}}{\pi L_{c}}\right)^{2} e^{-2 / \omega_{c}}\left[\frac{1}{\left(1-e^{-2 / \omega_{c}}\right)^{2}}\right. \\
\left.+\frac{2\left(1+e^{-4 / \omega_{c}}\right) \sin ^{2}\left(2 \pi \frac{\phi}{\phi_{0}}\right)-\left(1-e^{-2 / \omega_{c}}\right)^{2}}{\left\{4 e^{-2 / \omega_{c}} \sin ^{2}\left(2 \pi \frac{\phi}{\phi_{0}}\right)+\left(1-e^{-2 / \omega_{c}}\right)^{2}\right\}^{2}}\right],
\end{gathered}
$$

where $\omega_{c}=\sqrt{\alpha L_{c} / L_{0}}$. Therefore, the typical value of persistent current is given by

$$
I^{t y p}=\frac{2 \sqrt{2}}{\pi} \frac{e v_{F}}{L_{c}} \frac{e^{-1 / \omega_{c}}}{\left(1-e^{-2 / \omega_{c}}\right) \sqrt{1+e^{-2 / \omega_{c}}}} ; \quad T>T_{t h} .
$$

The typical persistent current [eq. (21)] scales as $I^{\text {typ }} \sim$ $k_{F} \ell$, which is in agreement with the result of von Oppen and Riedel's dimensipal analysis.12 In the case of integrable systems,12,13,23 on the other hand, it scales as $I^{t y p} \sim\left(k_{F} \ell\right)^{3 / 2}$. This mean that the persistent current of integrable billiards is much larger than that of chaotic ones.

As in the case of diffusive 3 and integrable 3 systems, $I^{(1)}$ gives a vanishing contribution to the grand canonical persistent current of an ensemble of billiards with different sizes or electron fillings as soon as the dispersion in $k_{F} \ell$ is order of $2 \pi$. Therefore we need to go to the canonical term $\Delta F^{(2)}$ in the free-energy expansion. Using eqs. (1) and (11), for the canonical persistent current one finds

$$
\begin{aligned}
& I^{(2)}(\phi)=-\frac{e \hbar}{2 \pi m A} \sum_{s, s^{\prime}} \frac{R_{T}\left(L_{s}\right) R_{T}\left(L_{s^{\prime}}\right)}{\sqrt{\left|\operatorname{det}\left(M_{s}-I\right)\right|\left|\operatorname{det}\left(M_{s^{\prime}}-I\right)\right|}} \\
& \times\left[\left(\omega_{s}+\omega_{s^{\prime}}\right) \sin \left\{\frac{\bar{S}_{s}+\bar{S}_{s^{\prime}}}{\hbar}+2 \pi\left(\omega_{s}+\omega_{s^{\prime}}\right) \frac{\phi}{\phi_{0}}\right\}\right. \\
& \left.-\left(\omega_{s}-\omega_{s^{\prime}}\right) \sin \left\{\frac{\bar{S}_{s}-\bar{S}_{s^{\prime}}}{\hbar}+2 \pi\left(\omega_{s}-\omega_{s^{\prime}}\right) \frac{\phi}{\phi_{0}}\right\}\right], \quad(22)
\end{aligned}
$$

where $\bar{S}_{s}=S_{s}-\hbar \sigma_{s} \pi / 2$. For an ensemble with a large dispersion of sizes or $k_{F}$, only the Cooperon term $\left(s^{\prime}\right.$ is $s$ time reversed) survives the $k_{F}$ average, and we obtain

$$
\begin{aligned}
\langle I(\phi)\rangle & \approx\left\langle I^{(2)}(\phi)\right\rangle \\
& =\frac{e \hbar}{\pi m A} \sum_{s} \frac{R_{T}^{2}\left(L_{s}\right) \omega_{s}}{\left|\operatorname{det}\left(M_{s}-I\right)\right|} \sin \left(4 \pi \omega_{s} \frac{\phi}{\phi_{0}}\right) .
\end{aligned}
$$

By using the H-OdA sum rule and the Gaussian winding number distribution, one obtains the average persistent current as

$$
\langle I(\phi)\rangle=\frac{\sqrt{2} e \hbar}{\pi^{3 / 2} m A \omega_{c}} \sum_{n=1}^{\infty} f\left(\omega_{c} ; n\right) n \sin \left(4 \pi n \frac{\phi}{\phi_{0}}\right),
$$

where the function $f\left(\omega_{c} ; n\right)$ is defined as

$$
f\left(\omega_{c} ; n\right)=\int_{0}^{\infty} d x \frac{\sqrt{x}}{\sinh ^{2} x} \exp \left(-\frac{n^{2}}{2 \omega_{c}^{2} x}\right) .
$$

Using the asymptotic expression $\sinh x \approx e^{x} / 2$ valid in high-temperature regimes, $x=T / T_{c}>1$, this integral can be performed analytically. The average persistent current for high temperature regime is thus given by

$$
\begin{aligned}
\langle I(\phi)\rangle=\frac{e \hbar}{\pi m A \omega_{c}} & \sum_{n=1}^{\infty}\left(1+\frac{2}{\omega_{c}} n\right) e^{-\frac{2}{\omega_{c}} n} \\
& \times \sin \left(4 \pi n \frac{\phi}{\phi_{0}}\right) ; \quad T>T_{t h} .
\end{aligned}
$$

$\langle I(\phi)\rangle$ shows the halving of the flux period with respect to the nonaveraged persistent current $I(\phi)$, i.e., eq. (14), characteristic of ensemble results. Moreover the amplitude of $\langle I\rangle$ for chaotic systems is positive, and scales as $\langle I\rangle \sim\left(k_{F} \ell\right)^{0}$, wich is much smaller than that of integrable systems, $13\langle I\rangle \sim k_{F} \ell$. Note that $\langle I(\phi)\rangle$ decreases rapidly with increasing temperature, and the higher harmonics die out more quickly.

In conclusion, we have studied the persistent current in quantum chaotic systems by using the Gutzwiller trace formula. We derived semiclassical formulas of a typical persistent current $I^{t y p}$ for single billiards and the averaged persistent current $\langle I\rangle$ for an ensemble of billiards at finite temperature. Moreover, we have shown that the persistent current amplitude scales as $I^{t y p} \sim k_{F} \ell$ and $\langle I\rangle \sim\left(k_{F} \ell\right)^{0}$. The former result coincides with von Oppen and Riedel's dimensional analysis.12 The different $k_{F}$ dependence of persistent current between integrable $\left(I^{\text {typ }} \sim\left(k_{F} \ell\right)^{3 / 2}\right.$ and $\left.\langle I\rangle \sim k_{F} \ell\right)$ and chaotic systems is attributed to the different contributions from nonisolated and from isolated periodic orbits. Therefore, we can conclude that the quantum persistent current in ballistic systems becomes a tool to distinguish chaotic and regular classical dynamics experimentally. Unfortupately no experiment is yet available for chaotic billiards.24 We hope our results will encourage future experiment.

I would like to thank B. Friedman, Y. Takane, and H. Tamura for valuable discussions and comments. 
${ }^{1}$ For reviews of persistent current in mesoscopic systems, see H.F. Cheung, Y. Gefen and E.K. Riedel, IBM J. Res. Dev. 32, 359 (1988), U. Eckern and P. Schwab, Adv. Phys. 44, 387 (1995) and Y. Imry, Introduction to Mesoscopic Physics (Oxford University Press, New York, 1997) Chap. 4.

${ }^{2}$ H.F. Cheung, E.K. Riedel and Y. Gefen, Phys. Rev. Lett. 62, (1989) 587.

${ }^{3}$ H. Bouchiat and G. Montambaux, J. Phys. (Paris) 50, (1989) 2695.

${ }^{4}$ F. von Oppen and E.K. Riedel, Phys. Rev. Lett. 66, (1991) 84 .

${ }^{5}$ B.L. Altshuler, Y. Gefen and Y. Imry, Phys. Rev. Lett. 66, (1991) 88.

${ }^{6}$ N. Argaman, Y. Imry and U. Smilansky, Phys. Rev. B 47, (1993) 4440

${ }^{7}$ A. Szafer and B.L. Altshuler, Phys. Rev. Lett. 70, (1993) 587.

${ }^{8}$ V. Ambegaokar and U. Eckern, Phys. Rev. Lett. 65, (1990) 381 .

${ }^{9}$ A. Schmid, Phys. Rev. Lett. 66, (1991) 80.

${ }^{10}$ D. Ullmo, K. Richter, H.U. Baranger, F. von Oppen and R.A. Jalabert, Physica E 1, (1998) 268.

${ }^{11}$ For a review of quantum chaos in mesoscopic systems, see Chaos and Quantum Transport in Mesoscopic Cosmos, edited by K. Nakamura, Chaos Solitons Fractals 8 (1997) No.7/8.

${ }^{12}$ F. von Oppen and E.K. Riedel, Phys. Rev. B 48, (1993) 9170 .

${ }^{13}$ K. Richter, D. Ullmo and R.A. Jalabert, Phys. Reps. 276, (1996) 1.

${ }^{14}$ M.V. Berry and M. Tabor, Proc. R. Soc. Lond. A. 349, (1976) 101.

${ }^{15}$ M.V. Berry and M. Tabor, J. Phys. A 10, (1977) 371.

${ }^{16}$ M.C. Gutzwiller, in Chaos and Quantum Physics, edited by M.-J. Giannoni, A. Voros and J. Zinn-Justin (Elsevier, Amsterdam, 1991) p. 201.

17 J.H. Hannay and A.M. Ozorio de Almeida, J. Phys. A 17, (1984) 3429.

18 M.V. Berry and M. Robnik, J. Phys. A 19, (1986) 649.

19 M.V. Berry and J.P. Keating, J. Phys. A 27, (1994) 6167.

${ }^{20}$ At low temperature $T \ll \Delta / k_{B}$, where $\Delta \ll k_{B} T_{t h}$, longer periodic orbits become more important and make a large contribution to the persistent current. In this limit the problem becomes nonperturbative, so that one must take into account off-diagonal terms between very long classical trajectories. Moreover in this limit the thermodynamical expansion [eq. (8)] breaks down.

${ }^{21}$ O. Agam, J. Phys. I France 4, (1994) 697.

${ }^{22}$ In this paper discussion will be confined to chaotic billiards for which the boundary is complicated enough (see Fig. 1) so that one may consider the corresponding set of the shortest periodic orbits as covering the phase space. Therefore we can use the H-OdA sum rule and the Gaussian winding number distribution even for the shortest periodic orbits.

${ }^{23}$ R.A. Jalabert, K. Richter and D. Ullmo, Surf. Sci. 361/362, (1996) 700.

${ }^{24}$ For an experimental study of the persistent current for integrable systems, see D. Mailly, C. Chapelier and A. Benoit, Phys. Rev. Lett. 70, (1993) 2020. 